\title{
Macrophage ferritin and iron deposition in the rat air pouch model of inflammatory synovitis
}

\author{
C J MORRIS, ${ }^{1}$ D R BLAKE, 'S D HEWITT, ' AND J LUNEC
}

From the ${ }^{1}$ Department of Rheumatology, The Medical School, University of Birmingham; and thg ${ }^{2}$ Department of Biochemistry, Selly Oak Hospital, Raddlebarn Road, Birmingham

SUMMARY Using a rat air pouch model of red cell promoted allergic inflammation, we havêّ investigated the relation between ferritin synthesis and iron deposition in pouch wall lining cell\$s These cells have structural and immunohistochemical similarities to human synovial intimal celis and studies of them are pertinent to the clinical situation. In control air pouch wall tissue aftem single or double antigenic challenge the (apo)ferritin containing macrophages are most numerous seven days after antigenic challenge when there is active connective tissue proliferation and generalised mononuclear cell response in the pouch wall, suggesting that (apo)ferritin produced in macrophages as part of the tissue inflammatory response. In contrast with contre tissue, where there is a steady decrease in positive cells over the ensuing weeks, injection of bloot into both single and double challenge air pouches produces a significant $(\mathrm{p}<0.001)$ an\& continuing rise in the numbers of ferritin containing macrophages after day 7 . Also, after 14 dayes Perls' positive ferric iron is detectable in increasing numbers of ferritin containing macrophage? a trend which is more marked in double challenge, blood injected air pouches. The histologiç data clearly show that there is a close relation between the presence of Perls' iron an proliferation of vascular and connective tissue elements in the pouch wall. We propose that th proinflammatory role of iron is the result of its ability to promote oxidative damage in tissues and discuss ways in which this may take place.

Biochemical and chemical evidence has led us to suggest that in rheumatoid arthritis deposits of iron in the synovial lining are linked with the chronicity of the inflammatory response ${ }^{12}$ probably because such iron catalyses the production of tissue hydroxyl radicals from superoxide and hydrogen peroxide. ${ }^{3}$ Pathological studies have shown that much of the iron is associated with the protein apoferritin, ${ }^{24}$ and that such ferritin is highly iron saturated, ${ }^{4}$ which in vitro has been shown to promote lipid peroxidation. ${ }^{5}$ If ferritin had this property in vivo it would cause membrane damage to lysosomes and release of potentially destructive hydrolytic enzymes. Our ultrastructural studies of a variety of synovitides show that intracellular iron saturated ferritin is associated with lysis of cytoplasm and organelles in synovial A cells only, iron containing $B$ cells being unaffected. ${ }^{6}$ Since $A$ cells are macrophage-like it may well be that intracytoplas-

Accepted for publication 1 October 1986.

Correspondence to Dr C J Morris, Rheumatism Research Wing, The Medical School, Birmingham B15 2TJ. mic damage in these cells is a refleccion of the ability to produce metabolically active oxygên metabolites with tissue destructive potential in the presence of iron. In macrophages, and certain epithelial cells, ingestion of erythrocytes is followed by their digestion in phagolysosomes and liberation of iron from haemoglobin, ${ }^{78}$ a process associated with increased ferritin production in the cytoplason and formation of aggregations in morphologicalfi distinct secondary lysosomes. ${ }^{910}$ This same process is likely to occur in the inflamed synovial membrame where repeated trauma causes minor bleeding from hyperaemic tissues, haemoglobin inducing synoviă cell ferritin formation in vitro ${ }^{11}$ and in the rabbit synovium in vivo. ${ }^{12}$

We have previously reported that whole bloळ.d when injected into an inflamed rat air pouch prolongs the inflammatory response. ${ }^{13}$ This proigflammatory or adjuvant effect was due to the read cells, but little effect was induced by the red ce्fil membrane. Using this model of red cell promoted allergic inflammation, we have extended our studies to examine the relation between (apo)ferritin synt 
esis, iron deposition, and the synovial tissue inflammatory response. This is pertinent to the clinical situation as we and others have found that the air pouch lining cells have many features in common with the human synovial membrane. ${ }^{14} 15$

\section{Materials and methods}

\section{ANIMAL MODEL}

An air pouch with allergic inflammation was induced in male Sprague-Dawley rats by the method previously described. ${ }^{15}$ Briefly, 16 groups of five rats were sensitised by injection of bovine serum albumin (BSA) in Freund's complete adjuvant on day -14 , a dorsal air pouch was created on day -1 , and on day 0 an inflammatory response was induced in the pouch by challenge with BSA in carboxymethylcellulose. A more permanent chronic inflammation was induced by rechallenge of the pouch on day +5 . A single fixed volume $(1 \mathrm{ml})$ of autologous whole blood, prepared as described previously, ${ }^{13}$ or $1 \mathrm{ml}$ saline control was injected into the pouch immediately after the antigenic challenge on day 0 (single challenge experiment -4 test and four control groups of rats) or +5 (antigenic rechallenge experiment -4 test and 4 control groups of rats).

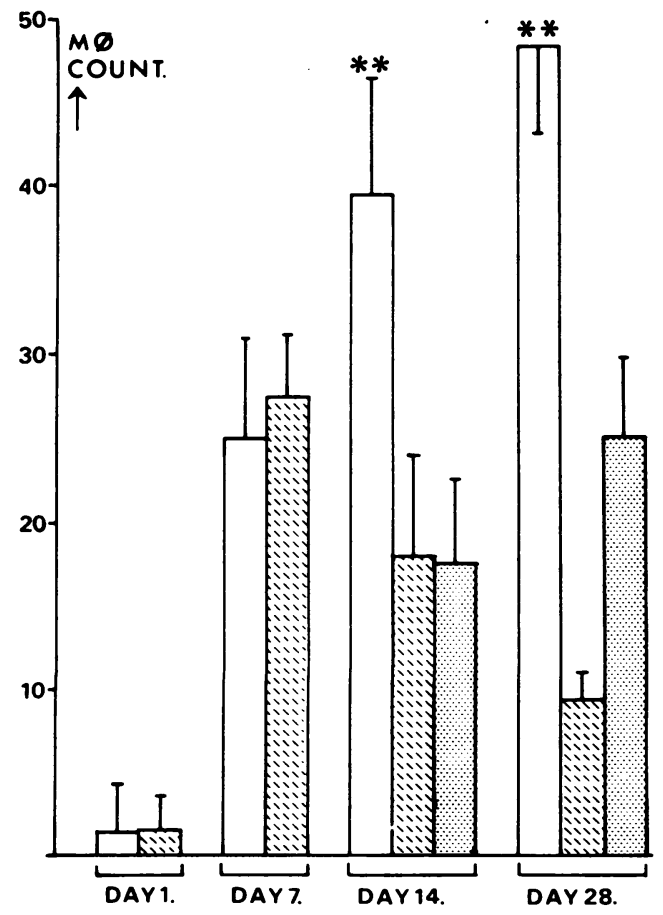

Fig. 1a
Animal groups were killed 1, 7, 14, and 28 days after blood or saline injection in both single challenge and rechallenge experiments.

\section{GHT M ICROS COPY}

Blocks of granulation tissue, removed at random from the pouch wall of each animal, were fixed in $10 \%$ buffered formol saline and embedded in paraffin wax by standard procedures. Serial sections were cut and stained by haematoxylin and eosin or haematoxylin and van Gieson for assessment of general tissue structure, and by Perls' prussian blue technique for ferric iron using acid hydrolysis to release ferric iron bound to proteins. Both muramidase (lysosome) and ferritin were demonstrated by standard peroxidase-antiperoxidase techniques. In the ferritin technique the antibody used had specificity for rat apoferritin.

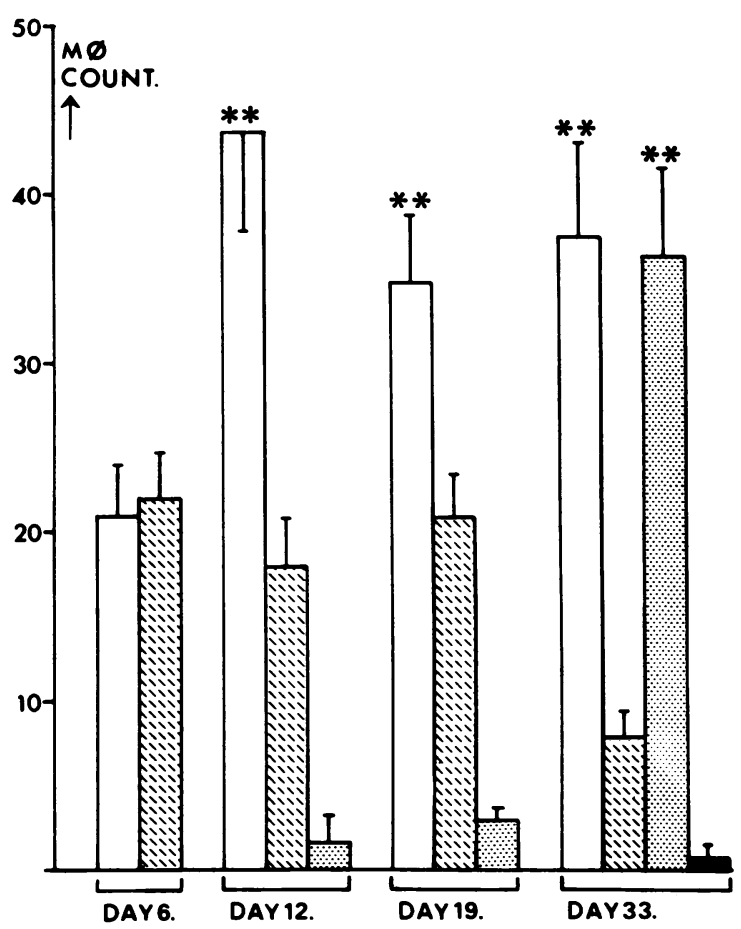

Fig. 1b

Fig. 1 Histogram of macrophage $(M \phi)$ counts from sections of rat air pouch walls after (a) a single BSA challenge, (b) a rechallenge with $B S A$. Open columns = ferritin positive macrophage counts from blood injected (test) tissue; cross hatched columns = ferritin positive macrophages from saline injected (control) tissue; stippled columns = Perls' positive macrophages, test tissue; filled in column = Perls' positive macrophages, control tissue. Bar lines = mean (SD). ${ }^{* *} p<0.001$. 


\section{CELL COUNTS}

The number of cells containing ferric iron, as shown by Perls' positive staining, and the number containing ferritin were counted in fields chosen at random from $5 \mu \mathrm{m}$ sections cut from control and test air pouch wall removed from rats killed at various times. Sections were viewed at a constant magnification $(\times 128)$, and the number of Perls' and ferritin positive cells were counted in random fields of view. The mean number of cells of each category per field and standard deviations were calculated for each time point in test and control air pouches, and the significance of the results was assessed using Student's $t$ test. Foamy macrophages containing carboxymethylcellulose ${ }^{15}$ were not included in the cell counts.

\section{Results}

SINGLE CHALLENGE (SC) A IR POUCH (Fig. 1a) All cells containing ferritin or iron, or both, in test and control pouches were also positive for muramidase (lysozyme) and were considered to be macrophages. In control (saline injected) pouch walls the number of ferritin containing macrophages was very low on day 1 but had increased significantly $(p<0.001)$ by day 7 . This time point coincides with

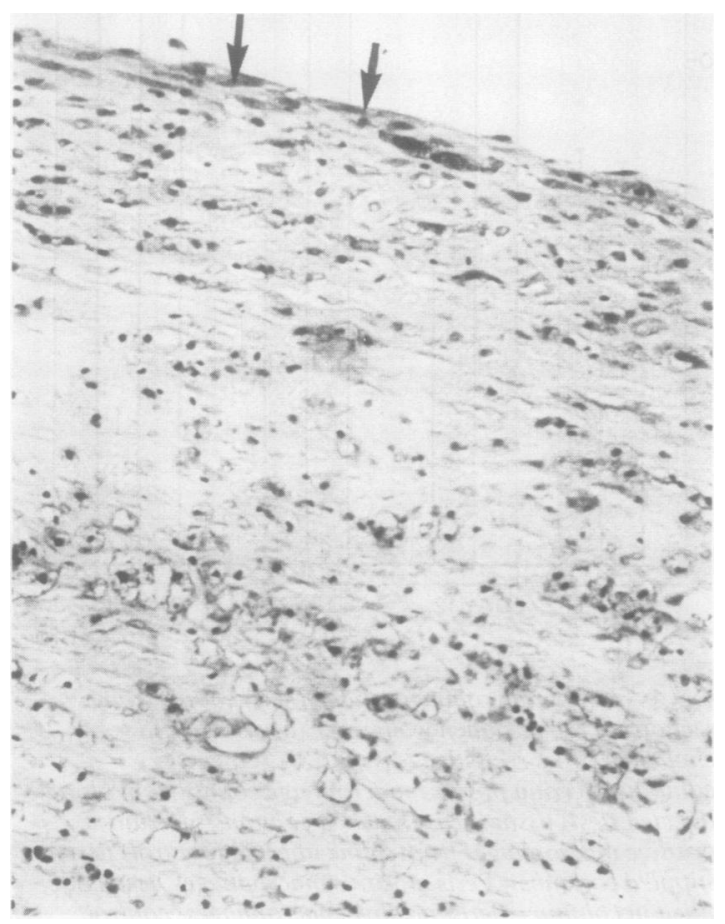

Fig. 2a active connective tissue proliferation and a gener康ised mononuclear cell response in the pouch wall ferritin being produced as part of the tissue imflammatory response. This is confirmed by the subsequent decrease in ferritin positive cells on daqs 14 and 28 when the inflammatory response resolving. Numbers of ferritin positive macrophags in test pouches injected with blood showed no significant difference from controls on days 1 and $\%$, but on days 14 and 28 there was a significant $(p<0.001)$ and continuing rise in ferritin positive cells in test animals when such cells in control tissues were decreasing. The test pouch walls also contained Perls' positive cells on day 14 , the numbers increasing significantly $(\mathrm{p}<0.01)$ between days $\mathrm{P}_{4}$ and 28 , when there was codistribution of ferritin and iron in some $50 \%$ of cells counted. Perls' positis cells were always positive for ferritin. The conneo-

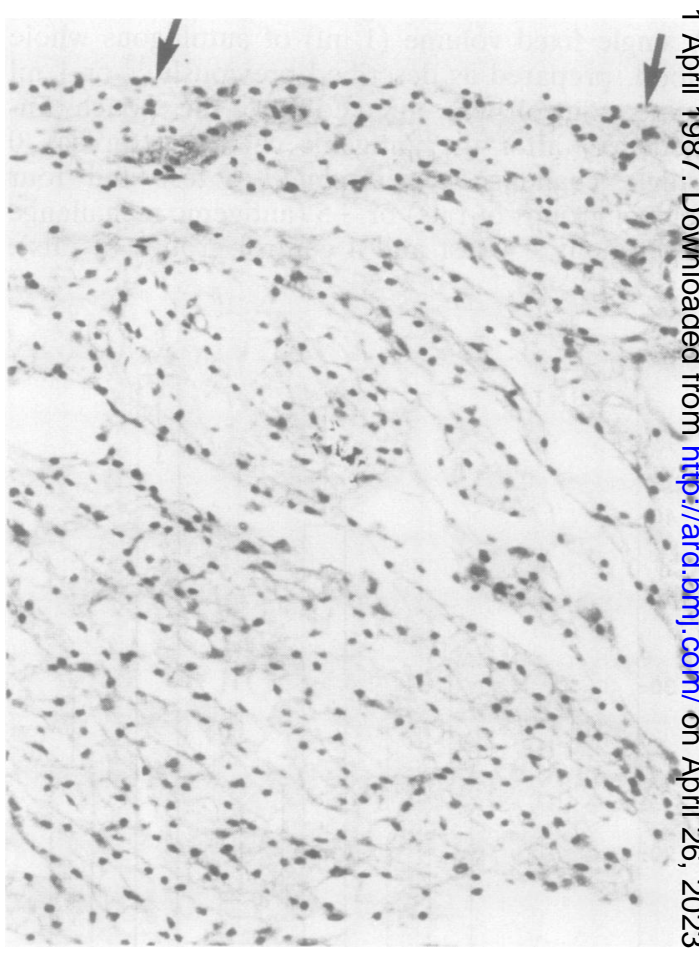

Fig. 2b

Fig. 2 (a) Section of blood injected pouch wall, day 28, after single BSA challenge. There is a well defined lining ç्] layer consisting mainly of fibroblasts (arrows) beneath which is well organised proliferating connective tissue containing a mixed inflammatory cell infiltrate (haematoxylin and eosin). (b) Section of control, saline injected pouch wall, day 28 , after single BSA challenge. T connective tissue response is minimal at this stage and the lining cell layer (arrows) is poorly defined (haematoxylin and eosin). 
tive tissue continued to evolve throughout the experiment (Fig. 2). Occasional Perls' positive cells were noted in control tissues, but numbers were too low for meaningful results to be obtained.

RECHALlenged (DC) A I POUCH (Fig. 1b) Counts of ferritin containing macrophages were found to be similar to those in SC pouches, with a significant rise in numbers $(\mathrm{p}<0.001)$ between days 6 and 12 . In contrast with SC experiments, however, the ferritin counts remained high for a further seven days before falling to the low level seen on day 33 . As in SC pouches, this ferritin response was linked with connective tissue proliferation. ${ }^{15}$ Ferritin counts from blood injected test pouches were higher than controls at all times and significantly so $(p<0.001)$ on days 12,19 , and 33. Cells containing both ferric iron and ferritin were present by day 12 , numbers increasing significantly between days 19 and $33(p<0.001)$, when most macrophages containing ferritin were also Perls' positive. At this time a few control pouch macrophages were also Perls' positive, though numbers were significantly $(p<0.001)$ lower than in the test pouches. In the later stages the blood injected pouch wall showed considerable vascular and connective tissue prolifer-

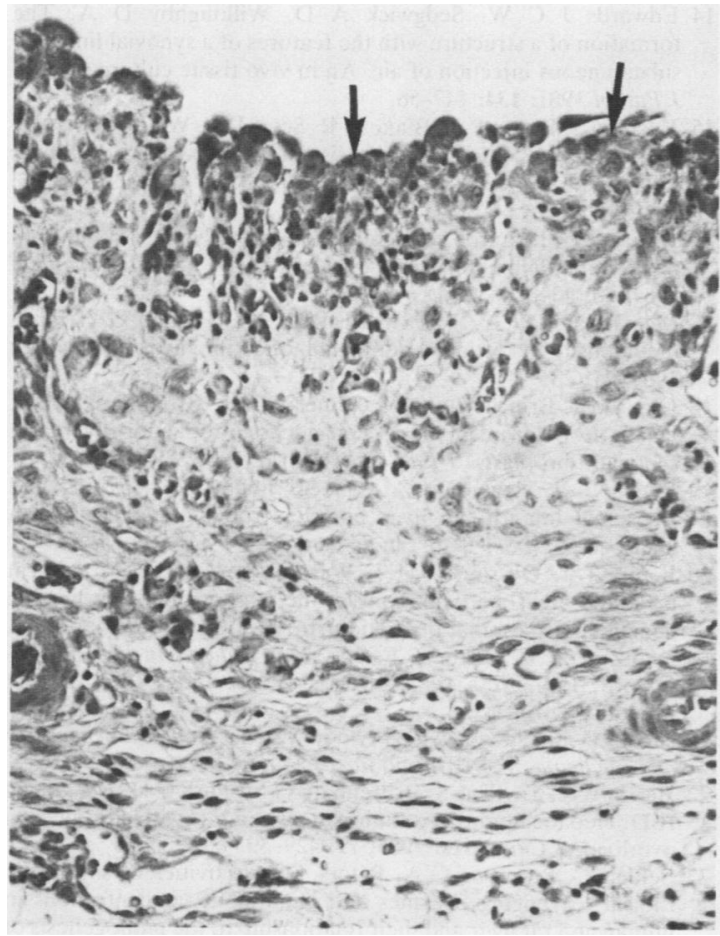

Fig. 3a

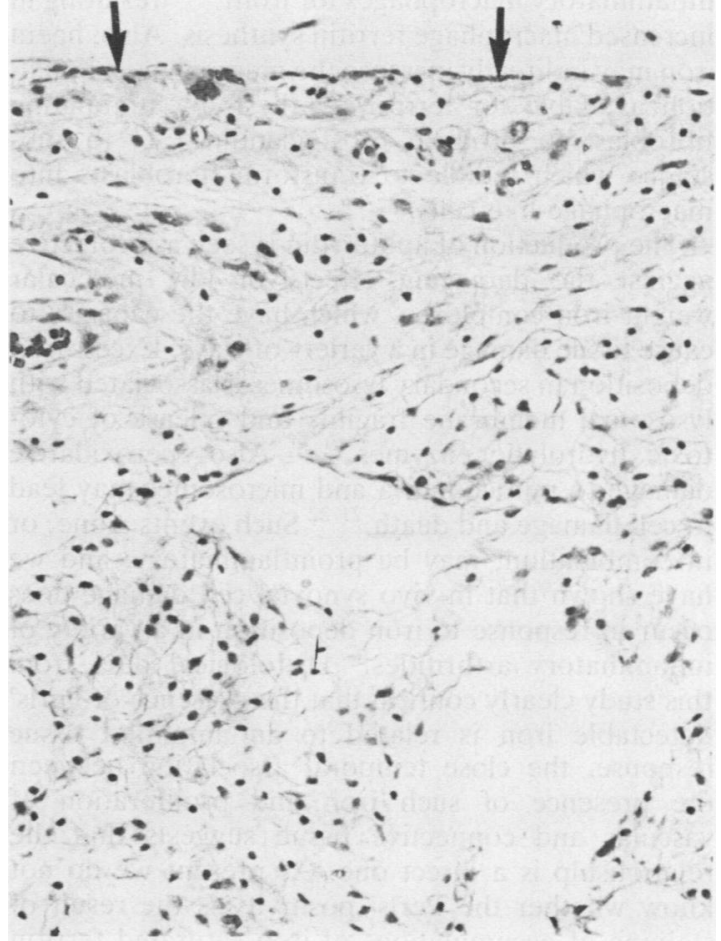

Fig. $3 b$

Fig. 3 (a) Section of blood injected pouch wall, day 33, after rechallenge with $B S A$. Note the hypercellularity of the lining cell layer (arrows) and the prominent connective tissue beneath (haematoxylin and eosin). (b) Section of control pouch wall, day 33. The thin lining cell layer is barely visible (arrows) and there is little connective tissue (haematoxylin and eosin).

ation and hypercellularity of the lining cell layer (Fig. 3).

\section{Discussion}

The macrophage apoferritin production after both single and double challenge clearly shows a close correlation with the development of the inflammatory response within the air pouch which we have previously described. ${ }^{15}$ This is in keeping with the observation of Birgegard and Caro that inflammatory macrophages have increased ferritin synthesis ${ }^{16}$ and indicates that inflammation rather than iron may be the inducer of apoferritin synthesis. We have already shown that haemoglobin is a major proinflammatory factor in blood injected into the air pouch, ${ }^{13}$ and in the absence of a similar response in control tissue this substance also causes the increase in ferritin synthesising pouch wall macrophages. Such a response is probably due in part to the local iron overload combined with increased avidity of 
inflammatory macrophages for iron.$^{16}{ }^{17}$ resulting in increased macrophage ferritin synthesis. Also, haem iron may indirectly increase the macrophage population available for ferritin synthesis by stimulating fibroblast to produce prostaglandin $E_{2},{ }^{18}$ a substance which is able to transform fibroblasts into macrophage-like cells.

The production of apoferritin is seen as protective against the damaging effects of low molecular weight iron complexes, which have the capacity to cause tissue damage in a variety of ways. Excess iron deposition in secondary lysosomes is associated with lysosomal membrane fragility and release of cytotoxic hydrolytic enzymes. ${ }^{19} 210$ Also, peroxidative damage to mitochondria and microsomes may lead to cell damage and death. ${ }^{21}{ }^{22}$ Such events alone, or in combination, may be proinflammatory, and we have shown that in vivo synovial cell damage does occur in response to iron deposition in a variety of inflammatory arthritides." Histological data from this study clearly confirm that the presence of Perls' detectable iron is related to an amplified tissue response, the close temporal association between the presence of such iron and proliferation of vascular and connective tissue suggests that the relationship is a direct one. At present we do not know whether the Perls' positivity is the result of staining of accumulations of iron saturated ferritin within secondary lysosomes, or of haemosiderin. The latter is derived from ferritin while under free radical attack, presumably catalysed by iron, when it is believed that there is loss of tyrosine residues and initiation of ferritin peptide degradation..$^{21}$ Selden $e t$ al have made sequential studies of patients accumulating or being depleted of iron which show that tissue damage is related to haemosiderin increase rather than to the increase in tissue ferritin. ${ }^{23}$ This would suggest that haemosiderin deposition occurs when iron promoted oxidation damage has already taken place and represents an end phase in the tissue response, the haemosiderin being relatively inert despite its high iron content. This is confirmed by our $x$ ray microanalytical studies of haemophilic synovitis, ${ }^{4}$ where haemosiderin is found in cells deep in the synovial intima and ferritin in proliferating cells at the synovial surface. Studies of synovial iron in rheumatoid arthritis also show that Perls' iron deposition does not correlate with indices of disease activity but is associated with persistent disease. ${ }^{2}$

\section{References}

1 Blake D R, Hall N D, Bacon P A, Dieppe P A, Halliwell B, Gutteridge $J \mathbf{M}$. The importance of iron in rheumatoid disease. Lancet 1981; ii: 1142-4.

2 Blake D R, Gallagher P J, Potter A R, Ball M J, Bacon P A. The effect of synovial iron on the progression of rheumatoid disease: a histologic assessment of patients with early rheumatoid synovitis. Arthritis Rheum 1984: 27: 495-501.
3 Halliwell B. The biological effect of the superoxide radical an its products. Bull Eur Phvsiopathol Respir 1981: 17: 2-28.

4 Morris C J. Wainwright A C. Steven M M. Blake D R. TI nature of iron deposits in hacmophilic synovitis: an immun chemical. ultrastructural and X-ray microanalytical studQ Virchows Arch /Cell Patholl 1984: 404: 75-85.

5 Gutteridge J M. Halliwell B. Treffry A. Harrison P M. Bla D R. Effect of ferritin containing fractions with different ir loading on lipid peroxidation. Biochem J 1983: 209: 557-60). @

6 Morris C J. Blake D R. Wainwright A C. Steven M Relationship between iron deposits and tissue damage in the synovium: an ultrastructural study. Ann Rheum Dis 1986: $4 \overrightarrow{5}$ 21-6.

7 Fedorko M E. Cross N L. Hirsch J G. Appearance a d distribution of ferritin in mouse peritoneal macrophages in vit@ after uptake of heterologous erythrocytes. J Cell Biol 1973; 59 289-305.

8 Zeligs $J$ D. Ultrastructure of the degradation of erythrocytes thyroid epithelial cells in vivo. Am J Pathol 1977; 89: 85-104

9 Chu L L H. Fineberg R A. On the mechanism of iron-induceid synthesis of apoferritin in the La cells. J Biol Chem 1969: 2499? $3847-54$

10 Hoy T G. Jacobs A. Changes in the characteristics and distribution of ferritin in iron-loaded cell cultures. Biochem $t$ 1981: 193: 87-92

11 Muirden K D, Fraser J R E, Clarris B. Ferritin formation synovial cells exposed to haemoglobin in vitro. Ann Rheum $D_{t s}$ 1967: 26: 251-9.

12 Roy S, Ghadially F N. Synovial membrane in experimenta 99 produced chronic haemarthrosis. Ann Rheum Dis 1969; 28 : 402-13.

13 Yoshino S. Blake D R. Hewitt S. Morris C J. Bacon P A. Effer of blood on the activity and persistence of antigen induced inflammation in the rat air pouch. Ann Rheum Dis 1985: $485-90$.

14 Edwards J C W. Sedgwick A D. Willoughby D A. T通 formation of a structure with the features of a synovial lining subcutaneous injection of air. An in vivo tissue culture systern J Pathol 1981: 134: 147-56.

15 Yoshino S. Bacon P A. Blake D R. Scott D L. Wainwright A Walton $\mathrm{K} \mathrm{W}$. A model of persistent antigen-induced chroñ inflammation in the rat air-pouch. Br J Exp Pathol 1984: 65: 201-14.

16 Birgegard G. Caro J. Increased ferritin synthesis and irô uptake in inflammatory mouse macrophages. Scand J Haemagl 1984: 33: 43-8.

17 MacDonald R A. MacSween R N M. Pechet G S. Irê metabolism by reticuloendothelial cells in vitro. Lab Inve् 1969: 21: 236-45.

18 Okasaki I. Brinckerhoff C E. Sinclair P R, Bronkowsky H 올. Harris E D. Iron increases collagenase production by rab synovial fibroblasts. J Lab Clin Med 1981: 97: 396-402.

19 Peters T J. Seymour C A. Acid hydrolase activities a lysosomal integrity in liver biopsies from patients with iron overload. Clin Sci 1976; 50: 75-8.

20 Peters T J. O'Connell M J. Ward R J. Role of frec-radie mediated lipid peroxidation in the pathogenesis of hepaft damage by lysosomal disruption. In: Poli G. Checseman K $甘$. Dianzani M U. Slater T F, eds. Free radicals in liver injury. Oxford: IRL Press 1985: 107-15. (Proceedings Internatiofal Meeting. Turin.)

21 Jacobs A. In: Jacobs A. Worwood M. eds. Iron in biochemis and medicine. Vol II. London: Academic Press, 1980: 427-5\%

22 Bacon B R. Tavill A S. Brittenham G M. Park C H. Recknag R D. Hepatic lipid peroxidation in vivo in rats with chronic irs overload. J Clin Invest 1983; 71: 429-39.

23 Selden C. Seymour C A. Peters T J. Activities of some fre radical scavenging enzymes and glutathione concentrations human and rat liver and their relationship to the pathogenesisef tissue damage in iron overload. Clin Sci 1980; 58: 211-9. 